\section{Producción científica sobre ameloblastomas en GoPubMed y SciELO}

\section{Scientific production on ameloblastomas in GoPubMed and SciELO}

\section{Resumen}

Objetivo: Describir la producción científica mundial sobre ameloblastomas indexada en GoPubMed y SciELO. Métodos: Se realizó un estudio observacional descriptivo transversal considerando como unidad de análisis los artículos publicados en revistas indexadas en GoPubMed y SciELO. Las redes de coautoría y co-ocurrencia de descriptores se crearon con el software VOSviewer y el método full counting. Resultados: En GoPubMed se recuperaron 4159 artículos con un promedio anual de publicación de 56,97 \pm 39,62. Existió predominio de los documentos publicados en el período 2010-2014 $(\mathrm{n}=758 ; 18,23 \%)$ procedentes de Estados Unidos $(\mathrm{n}=129 ; 3,1 \%)$ así como de artículos publicados en la revista Oral Surgery Oral Medicine and Oral Pathology $(\mathrm{n}=40 ; 0,96 \%)$. Los investigadores Hiroyuki Kumamoto y Chong Huat Siar constituyeron los autores con mayor número de artículos y redes de coautoría y cooperación internacional (36 y 31 respectivamente). «Ameloblastoma» y «mandibular neoplasms» fueron los descriptores con mayor número de ocurrencias. En SciELO se recuperaron 84 artículos con un promedio anual de publicación de 4,94 $\pm 2,88$. Existió predominio de artículos de autoría brasileña ( $\mathrm{n}=31 ; 36,9 \%)$, investigaciones originales $(\mathrm{n}=41 ; 48,81 \%)$ y documentos en idioma español $(\mathrm{n}=41 ; 48,81 \%)$. Conclusiones: Se evidenció una tendencia al incremento de las investigaciones en los últimos ańos, predominando los estudios originales así como la creación de redes internacionales de colaboración científica.

Palabras clave: Ameloblastoma; Indicadores bibliométricos; Investigación; Publicaciones; Revistas electrónicas.

\begin{abstract}
Objective: To describe the worldwide scientific production on ameloblastomas indexed in GoPubMed and SciELO databases. Methods: An observational, descriptive cross-sectional study was carried out, considering as a unit of analysis the articles published in journals indexed in GoPubMed and SciELO. The co-authorship and co-occurrence of descriptors networks were created with the VOSviewer software and the full counting method. Results: It was recovered 4159 articles from GoPubMed with an annual average of $56.97 \pm 39.62$. There was a predominance of documents published in the period 2010-2014 ( $\mathrm{n}=758 ; 18.23 \%)$ from the United States $(\mathrm{n}=129 ; 3.1 \%)$ as well as articles published in the journal Oral Surgery Oral Medicine and Oral Pathology ( $\mathrm{n}=40 ; 0.96 \%)$. The researchers Hiroyuki Kumamoto and Chong Huat Siar were the authors with the largest number of articles and networks of co-authorship and international cooperation
\end{abstract}

ISSN: 1560-9111

\section{Artículo Original}

Ibraín Enrique Corrales-Reyes ${ }^{1, a}$

1 Universidad de Ciencias Médicas de Granma. Clínica Estomatológica de Especialidades Manuel de Jesús Cedeño Infante. Bayamo, Cuba. ${ }^{a}$ Estudiante

\section{Correspondencia:}

Ibraín Enrique Corrales-Reyes

Correo electrónico: iecorralesr@infomed.sld.cu Ignacio Pérez \# 12A e/ Avenida 26 de Julio y Donato Mármol. CP: 87300. Jiguaní-Granma, Cuba.

Conflicto de intereses: El autor declara no tener conflictos de interés.

Fuente de financiamiento: Autofinanciado

Fecha de recepción: 24/01/18

Fecha de aceptación: 13/02/18 
(36 and 31 respectively). "Ameloblastoma» and "mandibular neoplasms» were the descriptors with the highest number of occurrences. In SciELO, 84 articles were recovered with an annual publication average of $4.94 \pm 2.88$. There was a predominance of articles of brazilian authorship $(n=31 ; 36.9 \%)$, original research $(n=41 ; 48.81 \%)$ and documents in Spanish $(n=41 ; 48.81 \%)$. Conclusions: There was a tendency to increase research in recent years, with original studies prevailing as well as the creation of international networks of scientific collaboration.

Keywords: Ameloblastoma; Bibliometric indicators; Electronic journals; Publications; Research.

\section{Introducción}

Los ameloblastomas son neoplasias odontogénicas benignas de comportamiento agresivo e infiltrante con una gran capacidad de recidiva y rara capacidad de metástasis ${ }^{1,2}$. Representan el $11 \%$ de todos los tumores odontogénicos y poseen un crecimiento lento y asintomático caracterizado por expansión de la cortical del hueso así como perforación e infiltración en los tejidos blandos ${ }^{3,4}$.

Fueron descritos por primera vez por Cusack en 1827 y el primer caso en la literatura científica fue presentado por Broca. Falkson completó la primera descripción histológica detallada en 1879. Posteriormente, en 1885 , Malassez introdujo el término adamantimoma-epitelioma. Desde entonces se han utilizado numerosos sinónimos para referirse a estos tumores benignos de los maxilares, hasta la actual denominación de ameloblastomas ${ }^{1}$.

Estas neoplasias se originan del epitelio odontogénico a partir de los componentes epiteliales del desarrollo dentario como son: restos de la lámina dental, epitelio reducido del esmalte, restos de Malassez y capas de células basales del epitelio superficial adyacente ${ }^{5}$. Se describen cuatro tipos de ameloblastomas benignos: sólido/multiquístico (SM), uniquístico, periférico y desmoplásico ${ }^{6}$. Los SM son los subtipos más comunes (aproximadamente el 80\% de los casos) y tienen predilección por las porciones posteriores de la mandíbula, particularmente el ángulo y la rama. A su vez, los desmoplásicos se presentan con mayor frecuencia en la región anterior de los maxilares.

Histológicamente pueden clasificarse en: folicular, plexiforme, acantomatoso, de células granulosas, desmoplásico y células basales, siendo la forma folicular la más frecuente, seguida de la plexiforme. Radiológicamente suelen describirse como una lesión radiolúcida, con límites netos, corticalizada y expansiva que adelgaza o erosiona la cortical ósea. Se han descrito dos patrones radiológicos de esta lesión: unilocular y multilocular, este último pudiendo asemejar un panal de abejas ${ }^{1}$.

A pesar de dicha clasificación, no se ha descrito relación entre la edad, sexo, tipo histológico ni comportamiento o agresividad del tumor. La reabsorción radicular es altamente sugestiva de ameloblastoma. Los dientes relacionados con la lesión están vitales y posteriormente pueden tener migración, movilidad, reabsorción radicular y parestesia ${ }^{1}$. En adición, los ameloblastomas pueden causar desde una deformación local agresiva hasta una simetría facial afectando funciones importantes como la masticación, fonación y estética.
El diagnóstico definitivo debe estar basado en el estudio anatomopatológico, el cual puede ser mediante punción y aspiración con aguja fina o biopsia de la lesión ${ }^{2}$. El tratamiento de los ameloblastomas es quirúrgico e incluye intervenciones radicales como la resección marginal, en bloque o la resección segmentaria/hemisección ${ }^{1}$.

Considerando la magnitud de las afectaciones clínicas y psicológicas causadas a los pacientes por los ameloblastomas, las investigaciones, así como los artículos científicos resultantes de las mismas, aumentan anualmente. En este sentido, un análisis bibliométrico de la producción científica mundial indexada en importantes bases de datos indicaría las tendencias de las investigaciones en esta área del conocimiento. Dado lo anterior, el objetivo del presente estudio fue describir la producción científica sobre ameloblastomas indexada en GoPubMed y SciELO.

\section{Métodos}

Se realizó un estudio observacional, descriptivo y transversal mediante un análisis bibliométrico considerando como unidad de análisis los artículos sobre ameloblastomas publicados en las revistas indexadas en GoPubMed y Scientific Electronic Library Online (SciELO). No se utilizaron límites de tiempo, por lo que los períodos estudiados transcurren desde 1945 hasta mayo de 2017 y desde 1999 hasta mayo de 2017 en GoPubMed y SciELO respectivamente.

Se estudiaron las siguientes variables: año de publicación del artículo, país, revista, idioma, tipología documental (originales, casos clínicos, revisiones, reseña de libro y comunicación rápida), autoría, redes de colaboración científica y descriptores. Las contribuciones provenientes de los países fueron tomadas de las afiliaciones de los autores en los respectivos artículos.

La búsqueda y recuperación de la información se realizó el 25 de mayo de 2017 por medio de la estrategia "ameloblastoma” [All Fields]. Se consultó la herramienta de búsqueda, recuperación y análisis de información GoPubMed ${ }^{7}$, así como la base de datos SciELO ${ }^{8}$ (integrado). La búsqueda en SciELO fue realizada por término libre (en cualquier parte del texto). Como estas herramientas tienen cobertura de literatura y calidad diferentes, los resultados son presentados de manera independiente debido a que no es técnicamente posible integrarlos. 
Para la creación del mapeo bibliométrico de las redes de coautoría y co-ocurrencias de descriptores se empleó el software VOSviewer ${ }^{9}$ versión 1.6.5 haciendo uso del método full counting ${ }^{10}$. El empleo de este método permite que, por ejemplo, la autoría de un artículo publicado por tres investigadores sea asignada a cada uno de ellos con un peso similar en términos de colaboración científica.

Las redes de cooperación científica entre autores así como las co-ocurrencias de los descriptores consistieron en nodos o conglomerados y enlaces. Los nodos representaron a los autores o descriptores mientras que los enlaces conectaron a los autores o descriptores en forma de coautorías u co-ocurrencias respectivamente. Los datos obtenidos se exportaron a una base de datos (MS Excel, Microsoft Corp., EE. UU.) con la cual se realizó el análisis estadístico.

\section{Resultados}

En GoPubMed se recuperaron 4159 artículos con un promedio anual de publicación de 56,97 $\pm 39,62$. El año con mayor cantidad de artículos publicados fue el 2014 $(\mathrm{n}=187 ; 4,5 \%)$ mientras que el de menor producción fue $1945(n=2 ; 0,05 \%)$. En la figura 1 se distribuyen los artículos según quinquenio, encontrándose un promedio de publicación de 277,27 $\pm 175,91$. La mayor cantidad de artículos se publicó en el período 2010-2014 ( $\mathrm{n}=758 ; 18,23 \%)$; el quinquenio con menor cantidad de artículos fue 1945-1949 ( $n=42 ; 1,01 \%)$.

Se publicaron contribuciones provenientes de 66 países siendo Estados Unidos ( $\mathrm{n}=129 ; 3,1 \%)$, la India $(\mathrm{n}=111$; $2,67 \%)$ y Japón $(n=84 ; 2,02 \%)$ los de mayor cantidad de artículos. En la figura 2 se muestra la distribución de los 18 países con $\geq 10$ artículos, cuya producción científica representa el 16,32\% $(n=679)$ del total.

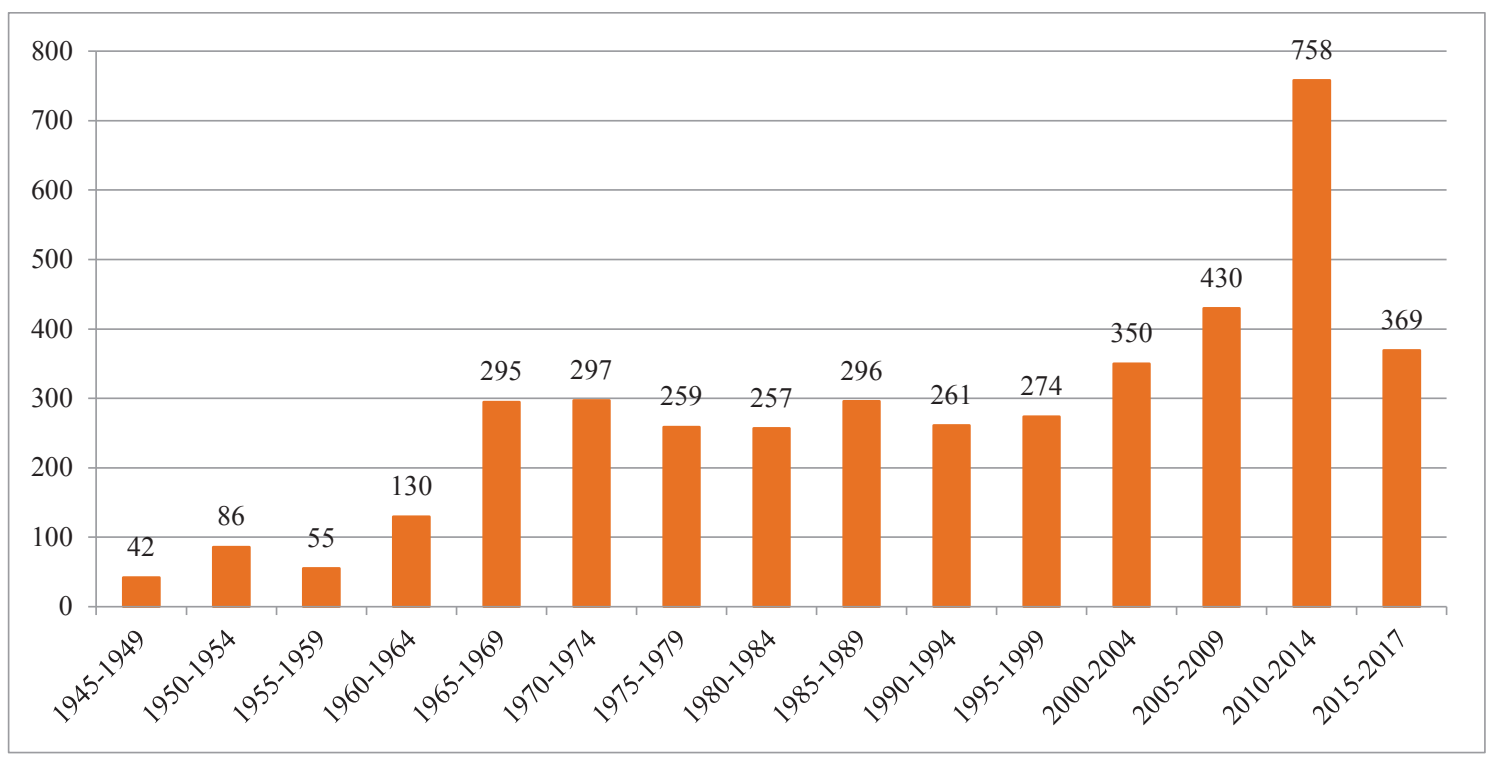

Figura 1. Distribución quinquenal de los artículos

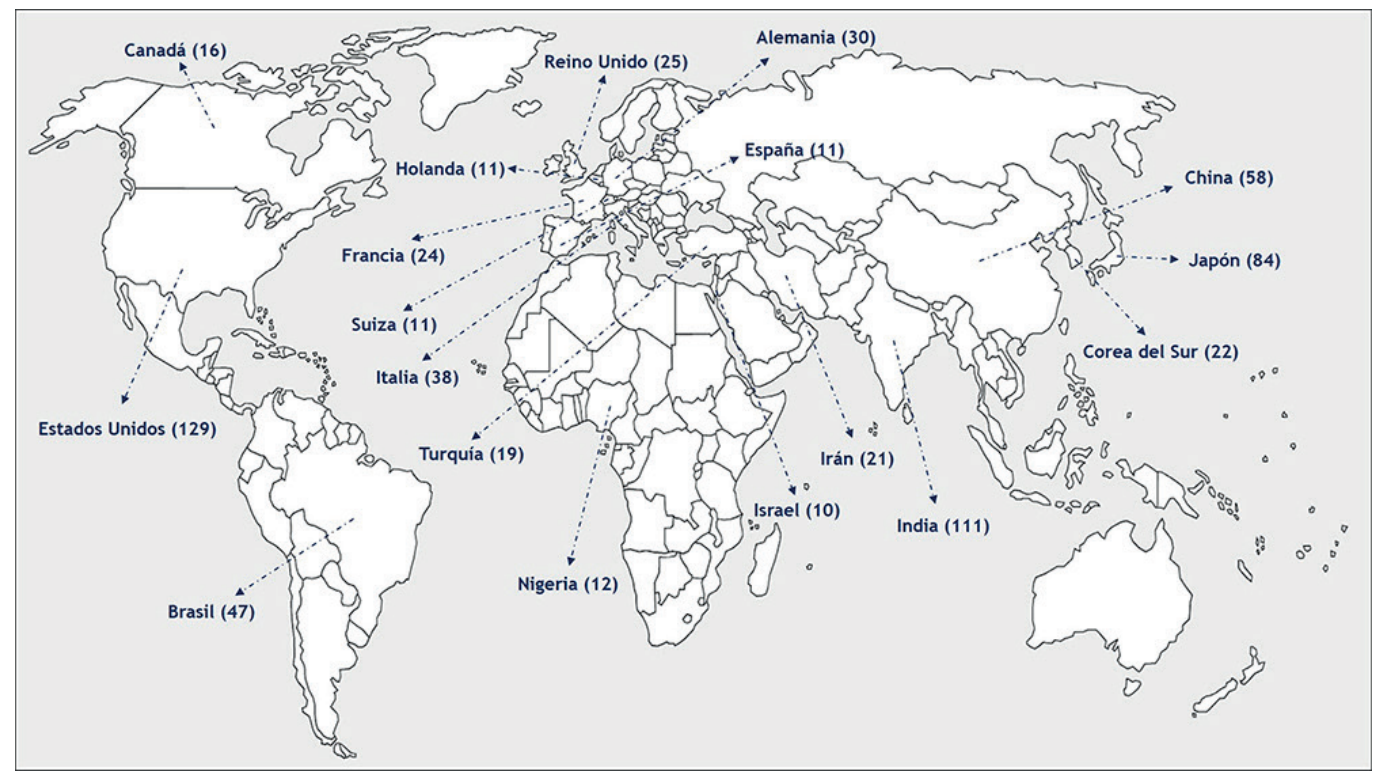

Figura 2. Distribución de los países con $\geq 10$ artículos 
Los artículos se publicaron en 576 revistas, siendo Oral Surgery Oral Medicine and Oral Pathology la publicación con mayor cantidad de documentos $(\mathrm{n}=40 ; 0,96 \%)$; a continuación se ubicó el Journal of Oral and Maxillofacial Surgery $(\mathrm{n}=32 ; 0,77 \%)$ y el Journal of Oral Pathology and Medicine $(\mathrm{n}=27 ; 0,65 \%)$. En la tabla 1 se muestra la distribución de las 13 revistas con $\geq 11$ artículos cuya producción científica representa el 5,70\% $(n=237)$ del total.

Tabla 1. Distribución de las revistas con $\geq 10$ artículos

\begin{tabular}{clc}
\hline $\mathbf{N}^{\circ}$ & \multicolumn{1}{c}{ Revista } & Artículos \\
\hline 1 & Oral Surgery Oral Medicine and Oral Pathology* & 40 \\
2 & Journal of Oral and Maxillofacial Surgery & 32 \\
3 & Journal of Oral Pathology and Medicine & 27 \\
4 & Journal of Clinical and Diagnostic Research & 17 \\
5 & $\begin{array}{l}\text { Revue de Stomatologie, de Chirurgie Maxillo-faciale et } \\
\text { de Chirurgie }\end{array}$ & 17 \\
6 & $\begin{array}{l}\text { Oral Surgery, Oral Medicine, Oral Pathology, Oral } \\
7\end{array}$ & Jadiology, and Endodontics \\
8 & Journal of Craniofacial Surgery & 15 \\
9 & Minerva Stomatologica & 14 \\
10 & Journal of Oral and Maxillofacial Pathology & 14 \\
11 & Cancer & 12 \\
12 & International Journal of Oral and Maxillofacial Surgery & 11 \\
\hline & British Journal of Oral and Maxillofacial Surgery & 11 \\
\hline & J & 12 \\
\hline
\end{tabular}

* La revista Oral Surgery Oral Medicine and Oral Pathology es la continuación de Oral Surgery, Oral Medicine, and Oral Pathology, Oral Radiology, and Endodontics.
Con respecto a las redes de coautoría y cooperación internacional, en la figura 3 se muestra un mapeo bibliométrico en el que se observa una red de coautoría de los 4159 artículos recuperados. De un total de 10 534 autores, se muestran los que poseen $\geq 2$ artículos en coautoría. Los autores se relacionan conformando 21 conglomerados, constituyendo los investigadores Hiroyuki Kumamoto y Chong Huat Siar los nodos centrales y con mayor número de artículos con 36 y 31 respectivamente.

En la figura 4 se muestra la red de co-ocurrencias de los descriptores. De un total de 2693 descriptores, se muestran los 500 con mayor cantidad de co-ocurrencias conformando seis clústers. "Ameloblastoma» y "mandibular neoplasms" constituyen los nodos centrales con 3478 y 1598 ocurrencias respectivamente.

En SciELO se recuperaron 84 artículos con un promedio anual de publicación de 4,94 $\pm 2,88$. En la figura 5 se muestra la distribución anual de los artículos, siendo el 2012 el ańo con mayor cantidad de papers $(\mathrm{n}=11$; $13,1 \%$ ); mientras que en el 2000 y el 2003 no se publicó ningún artículo.

Se publicaron contribuciones provenientes de 10 países, siendo Brasil $(\mathrm{n}=31 ; 36,9 \%)$, España $(\mathrm{n}=22$; $26,19 \%)$ y Cuba $(\mathrm{n}=11 ; 13,1 \%)$ los de mayor cantidad de artículos. (Figura 6).

Con respecto al idioma, se publicaron $41(48,81 \%)$ artículos en español, $36(42,86 \%)$ en inglés y 16 (19,05\%) en portugués. El 48,81\% de los artículos fueron originales $(n=41)$, el $42,86 \%$ casos clínicos $(n=36)$ y el $4,76 \%$ fueron revisiones $(\mathrm{n}=4)$. La distribución de los artículos según tipología se muestra en la figura 7.

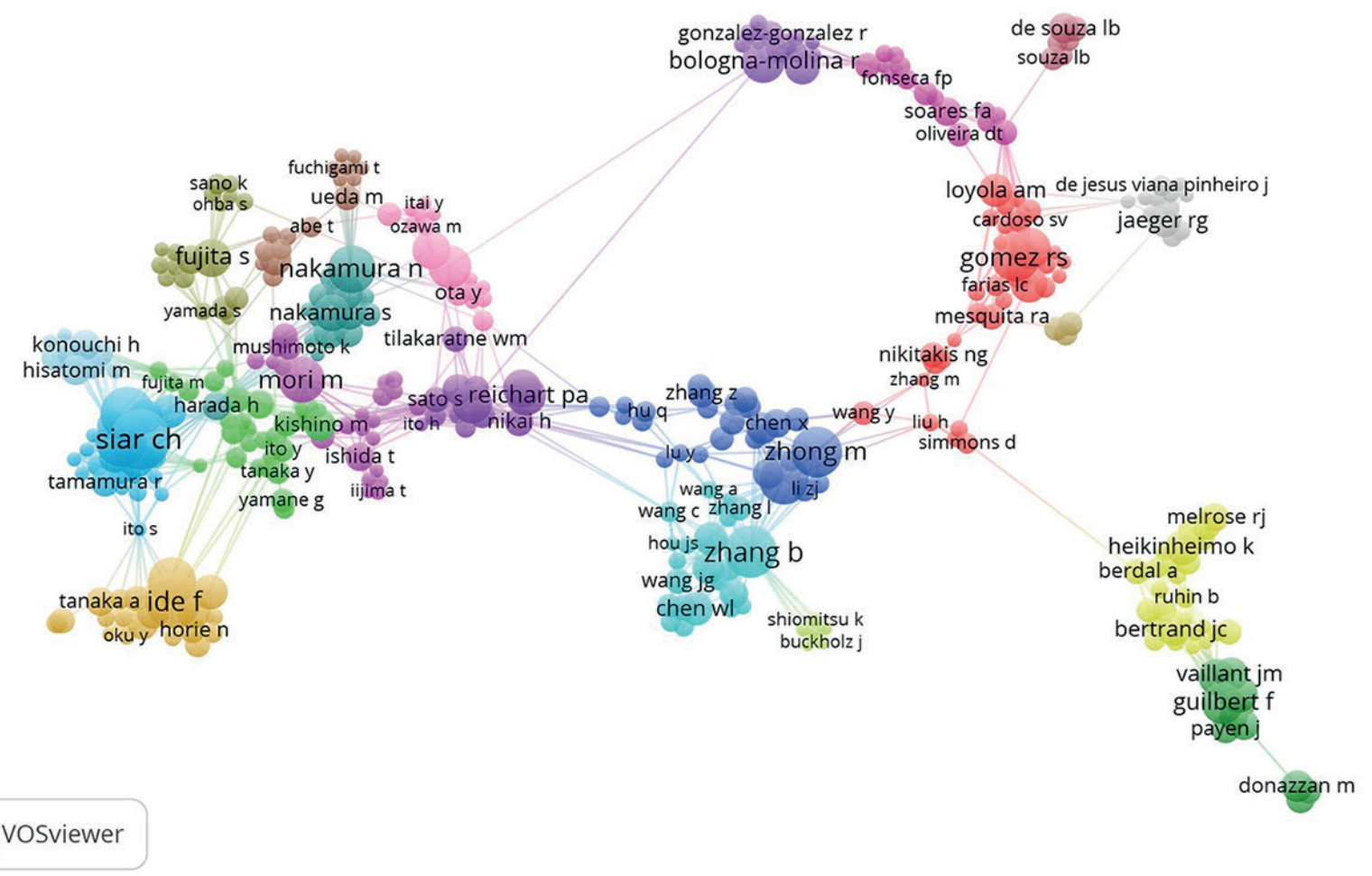

Figura 3. Red de co-ocurrencias de los autores con $\geq 2$ artículos publicados en coautoría 


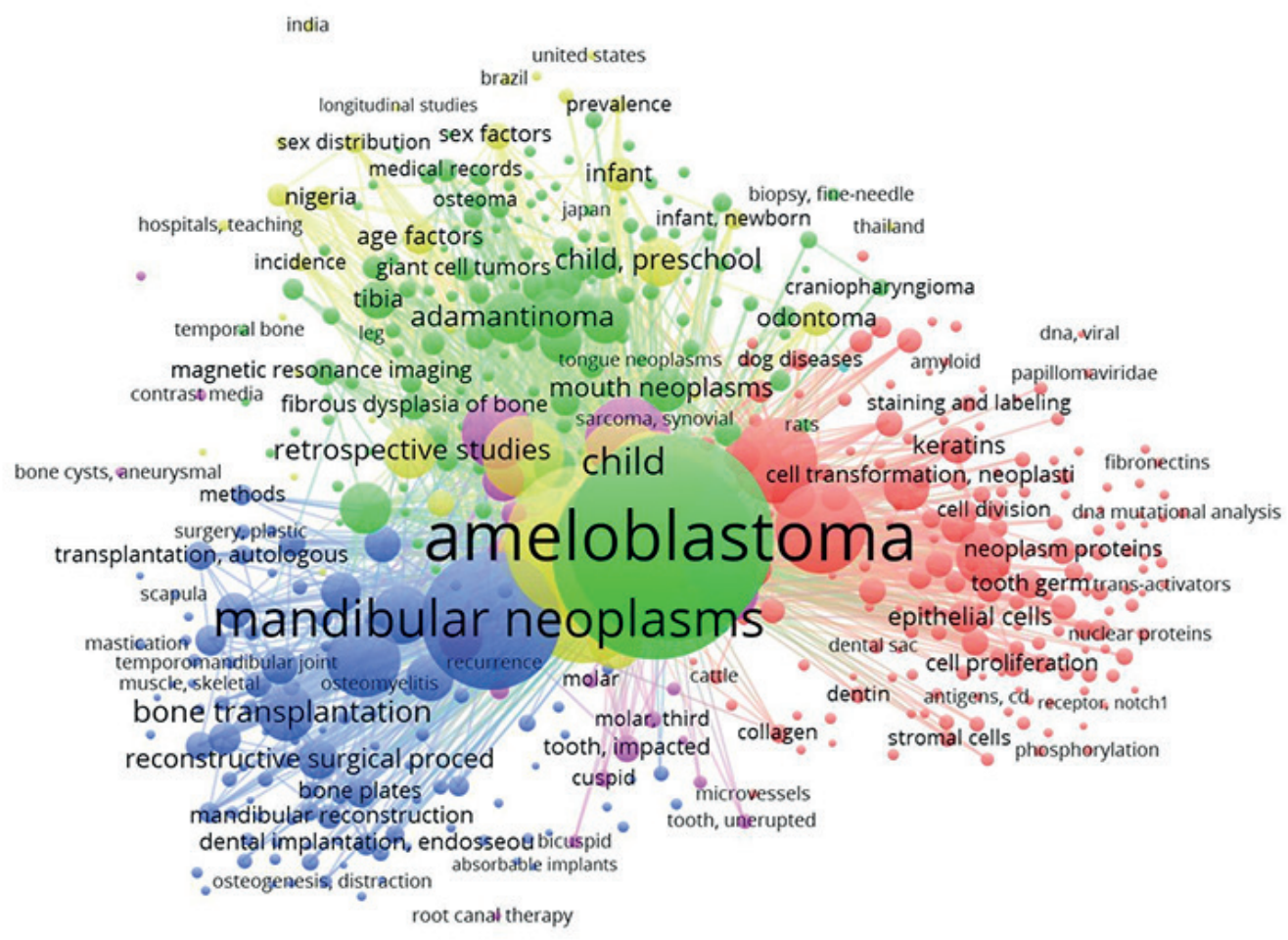

f. vosviewer

decompression, surgical

Figura 4. Red de co-ocurrencias de los descriptores

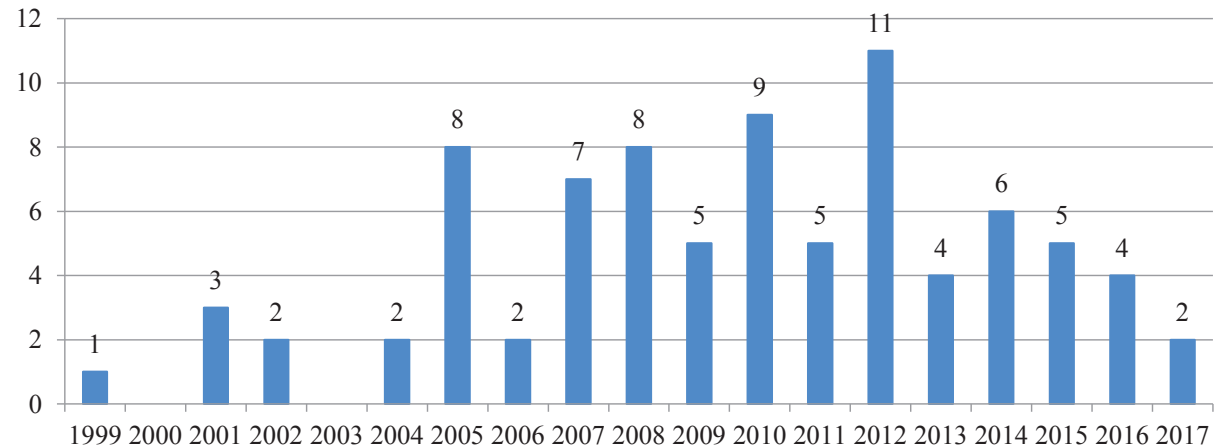

Figura 5. Distribución de los artículos indexados en SciELO según años de publicación

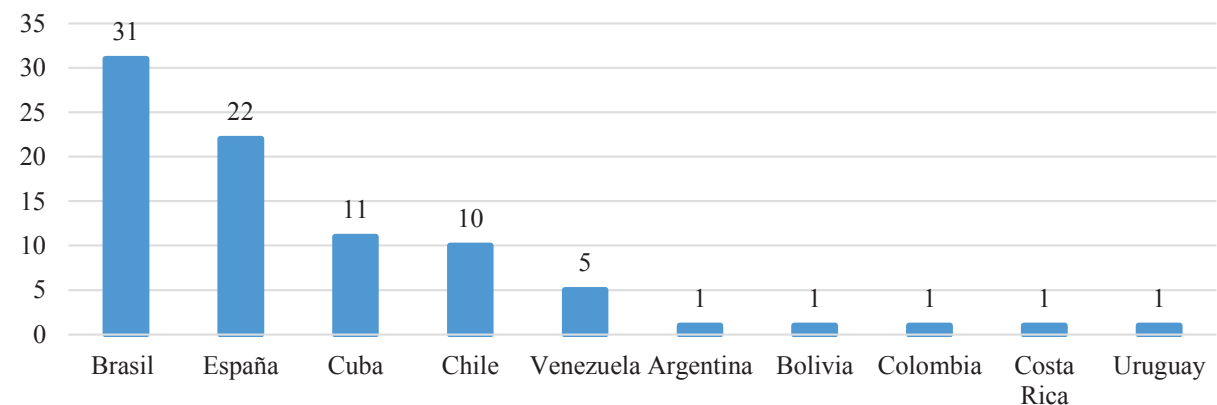

Figura 6. Distribución de los artículos indexados en SciELO según países 


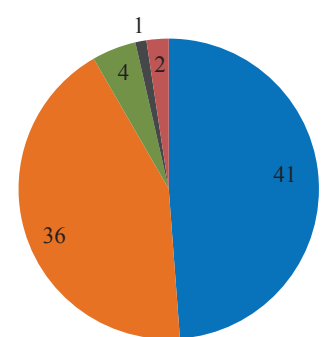

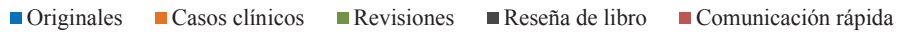

Figura 7. Distribución de los artículos indexados en SciELO según tipologías

\section{Discusión}

Los estudios bibliométricos han logrado un auge como recurso importante en la evaluación de la producción científica en un tema o área específica del conocimiento. Estos ofrecen una interesante visión del área que se estudia por lo que las instituciones académicas, los organismos de la financiación, las revistas y la comunidad científica en general se muestran cada vez más interesados en emplear estos análisis para evaluar la calidad de la investigación y la productividad de los investigadores 11,12 .

Para la realización del presente estudio se seleccionaron las herramientas GoPubMed y SciELO pues es reconocido que las revistas que indexan poseen calidad científica y rigor metodológico, lo cual avala en buena medida las investigaciones que publican. El mayor volumen de producción científica encontrado pertenece a GoPubMed debido a, entre otras razones, la mayor cobertura geográfica y tiempo de funcionamiento de este en comparación con SciELO.

Se encontró el liderazgo global de los Estados Unidos, la India y Japón en las investigaciones sobre estas neoplasias benignas (ameloblastomas) pues los artículos generados en estos países constituyeron el 7,79\% de la producción científica recuperada en GoPubMed. A escala iberoamericana, el liderazgo es asumido por Brasil, España y Cuba pues su producción científica representa el 76,19\% del total de artículos indexados en SciELO.

Estos resultados coinciden con lo reportado en el análisis bibliométrico de los 100 artículos sobre ameloblastomas más citados que se publicaron en el período 2000-2016 en el cual se encontró un predominio de documentos de autoría japonesa, brasileña y estadounidense a escala global ${ }^{13}$. Entre las razones subyacentes podríamos mencionar la presencia de un elevado número de investigadores, recursos y centros de investigación, así como a los financiamientos donados por fundaciones públicas a esta área del conocimiento como resultado del prestigio de la cirugía maxilofacial en esos países.

Existió una tendencia al incremento de las investigaciones y la publicación de artículos resultantes de las mismas en la última década, lo cual coincide con un estudio realizado en el área de la terapéutica farmacológica antimicrobiana ${ }^{14}$. Ello pudiera atribuirse al acelerado desarrollo de la ciencia y la técnica y su aplicación en las investigaciones biomédicas, donde cada año se realizan estudios con un nivel de profundidad mayor.

Algunas de las revistas con mayor número de artículos recuperados en GoPubMed se incluyen dentro de las publicaciones con mayor cantidad de artículos incluidos en los 100 más citados del período 2000-2016 según el análisis bibliométrico antes mencionado ${ }^{13}$. Tal es el caso de las revistas: Journal of Oral and Maxillofacial Surgery; Oral Surgery, Oral Medicine, Oral Pathology, Oral Radiology, and Endodontology; International Journal of Oral and Maxillofacial Surgery y British Journal of Oral and Maxillofacial Surgery.

Ello está dado por el hecho de que la publicación de una revista online, sea como forma exclusiva o sumada a su versión impresa, aumenta su visibilidad al facilitar el acceso a un ámbito de lectores mayor que el de los suscriptores de la versión impresa. Esto es más notorio si la versión electrónica se incluye en bases de datos de amplia difusión que permitan el acceso libre a los artículos, ya sea al título y el resumen en español e inglés o, además, a su texto in extenso ${ }^{15}$. Cuando miles de investigadores de todo el mundo buscan investigación actualizada sobre cirugía maxilofacial y áreas afines tienen un acceso directo a los artículos, lo cual aumenta las posibilidades de consumo y citación de la información ${ }^{16}$.

El predominio de investigaciones «originales» encontrado en SciELO coincide con el reportado en otras investigaciones ${ }^{17,18}$ y resulta comprensible si se tiene en cuenta que estos estudios poseen un elevado impacto al comunicar los resultados de investigaciones científicas relevantes. La segunda tipología más publicada fueron las presentaciones de casos por cuanto siempre resulta interesante divulgar los casos de entidades nosológicas poco frecuentes o cuyas presentaciones clínicas son atípicas. Ello difiere con lo reportado en investigaciones similares por Zyoud et al. ${ }^{17}$ y González-Alcaide et al. ${ }^{18}$ quienes encontraron que las cartas al editor y los artículos de revisión fueron las segundas tipologías más publicadas respectivamente. En el análisis realizado por Rodriguez-Morales et al. ${ }^{14}$ no se evaluó la producción científica registrada en esta base de datos según tipología. 
La publicación de un artículo por más de un autor refleja la formación de grupos de investigación, los cuales pueden evolucionar y convertirse en sólidos mecanismos e instancias de aprendizaje y actualización odontológica con investigaciones de mayor impacto científico. En este sentido, los investigadores Hiroyuki Kumamoto y Chong Huat Siar constituyeron los autores más productivos y con mayor número de redes de coautoría y cooperación internacional.

La colaboración entre los investigadores reviste gran importancia pues a medida que la ciencia ha evolucionado y las investigaciones se hacen cada vez más complejas y específicas, estos reconocen la importancia de las redes de colaboración y de los estudios multinacionales por lo que se relacionan con otros equipos de investigación como producto de ello. Además, es importante tener en cuenta que el crecimiento de la cooperación científica es un factor que influye positivamente en la atracción de citas, lo cual es favorable tanto para los autores como para las revistas ${ }^{19,20}$.

Uno de los retos actuales que enfrentan los profesionales de la salud es el manejo de la información disponible, tanto para su formación y actualización profesional como para resolver los desafíos médicos diarios. Indudablemente hoy en día se requiere de profesionales de la salud que tengan habilidades para la búsqueda y recuperación efectiva de información ${ }^{21}$. Los descriptores en ciencias de la salud no sólo son útiles para realizar una búsqueda, sino que además sirven para analizar los trabajos por la materia estudiada, permitiendo así descubrir la evolución de las corrientes investigadoras y los aspectos que interesan a los investigadores ${ }^{22}$. No debe subestimarse su trascendencia, pues se puede dificultar la difusión del documento e incluso su total olvido por problemas de identificación ${ }^{23}$.

El presente estudio posee limitaciones. Entre estas encontramos las relacionadas con las herramientas de búsqueda de información utilizadas, por cuanto no se pudo acceder a la producción científica indexada en la Web of Science y Scopus debido a los gastos de la suscripción. Además, no todas las investigaciones que se realizan sobre estas neoplasias de los maxilares se publican pues muchas de estas sólo se presentan en congresos o se publican en revistas no indexadas.

No obstante, el descriptor empleado en la estrategia de búsqueda coincide en español, inglés y portugués, con lo cual se eliminan los sesgos idiomáticos y se hace más incluyente el estudio. En adición, la calidad reconocida de las publicaciones incluidas en las bases de datos evaluadas y su cobertura fortalecen el hecho de que los documentos seleccionados constituyen una muestra representativa de la investigación internacional sobre ameloblastomas. Dado lo anterior, se recomienda realizar periódicamente este tipo de estudio con una frecuencia que bien pudiera ser quinquenal de manera que se obtuviera una panorámica actualizada de las características y tendencias de la producción científica en esta y otras áreas del conocimiento.
Se concluye que la producción científica sobre ameloblastomas muestra una tendencia al incremento de las investigaciones en los últimos años, predominando los estudios originales, la creación de redes internacionales de colaboración científica y demostrándose el liderazgo de los Estados Unidos, Japón, India y Brasil, lo cual evidencia la necesidad de incrementar la producción científica latinoamericana.

\section{Referencias bibliográficas}

1. López R, Jaeger F, Gomes JH, Leal RM. Ameloblastoma: un estudio retrospectivo de 48 casos. Rev Esp Cir Oral Maxilo Fac. 2013;35(4):145-49.

2. Hu S, Parker J, Divaris K, Padilla R, Murrah V, Wright JT. Ameloblastoma phenotypes reflected in distinct transcriptome profiles. Sci Rep. 2016;6:30867.

3. da Silva HE, Ramos ES, Quintáo AC, dos Santos PS. Ameloblastoma during pregnancy: a case report. J Med Case Rep. 2016;10:244.

4. Manimaran K, Sharma R, Sankaranarayanan S, Mahendra S. Regeneration of mandibular ameloblastoma defect with the help of autologous dental pulp stem cells and buccal pad of fat stromal vascular fraction. Ann Maxillofac Surg. 2016;6(1):97-100.

5. Orozco NI, Rodríguez LN, Ortiz F, Aburto MA, Compañ NK. Ameloblastoma. Reporte de un caso clínico en un paciente infantil. Odont Act. 2014;11(138):56-64.

6. Gardner D, Heikinheimo K, Shear M, Philipsen H, Coleman H. Ameloblastoma. In: World Health Organization classification of tumours: pathology and genetics of head and neck tumours. Lyon, France: International Agency for Research on Cancer; 2005.

7. GoPubMed. [Base de datos en Internet]. [accedido 25 May 2017]. Accesible en: http://gopubmed.org/web/gopubmed/

8. Scientific Electronic Library Online [SciELO integrado]. [Base de datos en Internet]. [accedido 25 May 2017]. Accesible en: http://www.scielo.org/php/index.php

9. Van Eck NJ, Waltman L. VOSviewer [Internet]. Leiden: Leiden University; 2010 [accedido 25 May 2017]. Accesible en: http://www.vosviewer.com

10. Perianes-Rodriguez A, Waltman L, Van Eck NJ. Constructing bibliometric networks: a comparison between full and fractional counting. J Informetrics. 2016;10:1178-95.

11. Eyre-Walker A, Stoletzki N. The assessment of science: the relative merits of post-publication review, the impact factor, and the number of citations. PLoS Biol. 2013;11:1001675.

12. Sinha Y, Iqbal FM, Spence JN, Richard B. A bibliometric analysis of the 100 most-cited articles in rhinoplasty. Plast Reconstr Surg Glob Open. 2016;4:820.

13. Corrales-Reyes IE, Reyes-Pérez JJ. Análisis bibliométrico de los 100 artículos sobre ameloblastomas más citados. Período 2000-2016. Educ Med. 2017 [accedido 25 May 2017]. Disponible en: http://dx.doi.org/10.1016/j. edumed.2017.03.013 [en prensa] 
14. Rodriguez-Morales AJ, Martinez-Pulgarin DF, Muñoz-Urbano M, Gómez-Suta D, Sánchez-Duque JA, Machado-Alba JE. Bibliometric assessment of the global scientific production of nitazoxanide. Cureus. 2017;9(5):e1204.

15. Reyes H, Bustos A, Andresen M, Palma J. La revista médica de Chile en el año 2012. Rev Méd Chile. 2013;141(8):1049-56.

16. Cartes-Velásquez R. Ingreso a LILACS, seguimos avanzando. J Oral Res. 2014;3(1):7-8.

17. Zyoud S, Al-Jabi SW, Sweileh WM. Worldwide research productivity in the field of electronic cigarette: a bibliometric analysis. BMC Public Health. 2014;14:667.

18. González-Alcaide G, Park J, Huamaní C, Belinchón I, Ramos JM. Evolution of Cooperation Patterns in Psoriasis Research: Co-Authorship Network Analysis of Papers in Medline (1942-2013). PLoS ONE. 2015;10(12):e0144837.
19. Boracci RA, Doval HC, Manente D, Tajer CD. Redes de colaboración científica en las publicaciones cardiológicas argentinas. Rev Argent Cardiol. 2009;77:487-92.

20. Corrales-Reyes IE. Co-authorship and scientific collaboration networks in Medwave. Medwave. 2017;17(9):7103.

21. Fernández-Altuna MÁ, Martínez A, Arriarán E, Gutiérrez D, Toriz HA, Lifshitz A. Uso de los MeSH: una guía práctica. Inv Ed Med. 2016;5(20):220-9.

22. Tomás-Casterá V, Sanz-Valero J, Wanden-Berghe C, Landaeta M. Descriptores versus palabras clave sobre nutrición: aportación a la correcta indización. An Venez Nutr. 2009;22(2):90-4.

23. de Granda JI, García F, Callol L. Importancia de las palabras clave en las búsquedas bibliográficas. Rev Esp Salud Pública. 2003;77:765-7. 